\title{
Nontuberculous Mycobacteria in Respiratory Tract Infections, Eastern Asia
}

\author{
Sami Simons, Jakko van Ingen, Po-Ren Hsueh, Nguyen Van Hung, P.N. Richard Dekhuijzen, \\ Martin J. Boeree, and Dick van Soolingen
}

To characterize the distribution of nontuberculous mycobacteria (NTM) species isolated from pulmonary samples from persons in Asia and their association with pulmonary infections, we reviewed the literature. Mycobacterium avium complex bacteria were most frequently isolated $(13 \%-81 \%)$ and were the most common cause of pulmonary NTM disease (43\%-81\%). Also pathogenic were rapidly growing mycobacteria ( $M$. chelonae, M. fortuitum, M. abscessus). Among all NTM isolated from pulmonary samples, 31\% $(582 / 1,744)$ were considered clinically relevant according to American Thoracic Society diagnostic criteria. Most patients were male (79\%) and had a history of tuberculosis (37\%). In Asia, high prevalence of rapidly growing mycobacteria and a history of tuberculosis are distinct characteristics of pulmonary NTM disease. This geographic variation is not well reflected in the American Thoracic Society criteria for NTM infections and could be incorporated in future guidelines.

$\mathrm{N}$ ontuberculous mycobacteria (NTM) are common in the environment and have been isolated worldwide (1). They are increasingly recognized as pathogens in humans. Pulmonary disease is the most common manifestation (2) and is thought to result from aerosol inhalation. Because of their omnipresence in the environment, isolation of NTM from the respiratory tract does not, per se, indicate NTM disease. Therefore, the American Thoracic Society (ATS)

Author affiliations: Radboud University Nijmegen Medical Centre, Nijmegen, the Netherlands (S. Simons, J. van Ingen, P.N.R. Dekhuijzen, M.J. Boeree); National Institute for Public Health and the Environment (RIVM), Bilthoven, the Netherlands (J. van Ingen, D. van Soolingen); National Taiwan University Hospital, Taipei, Taiwan (P.-R. Hsueh); and National Hospital of Tuberculosis and Respiratory Diseases, Hanoi, Vietnam (N.V. Hung)

DOI: 10.3201/eid1703100604 has established diagnostic criteria to help distinguish between contamination and true NTM disease (1).

Although NTM are present worldwide, much of the literature on pulmonary NTM disease comes from industrialized countries, mainly Europe, North America, and Japan. Differences in the NTM species distribution in clinical samples and disease have been noted among these regions (3); by extrapolation, these characteristics in other parts of the world probably differ as well.

Asia has a long history of NTM research, both clinical and fundamental. Tsukamura et al. have reported on pulmonary NTM infections in Japan dating back to the early 1970s (4). Yet because many studies were not reported in English, knowledge of the distribution of NTM species in Asia is limited. However, these data will enhance our understanding of NTM diversity between and within species and their association with NTM disease in humans. For this reason, we searched the literature on clinical NTM isolation and disease from different regions in Asia and compared our findings with previously published data from other regions.

\section{Literature Search}

From March 2009 to December 2009, we searched PubMed (www.ncbi.nlm.nih.gov/pubmed) for Englishlanguage articles about nontuberculous mycobacteria in Asia. The search strategy was as follows: "mycobacteria, atypical" [MeSH] AND "Asia" [MeSH] OR "atypical mycobacterium infections" [MeSH] AND "Asia" [MeSH]. We chose the term Asia to incorporate the following countries: Brunei, Cambodia, East Timor, Indonesia, Lao People's Democratic Republic, Malaysia, Myanmar, Philippines, Singapore, Thailand, Vietnam, Bangladesh, Bhutan, India, China, Hong Kong, Japan, South Korea, Mongolia, and Taiwan. 
We found 256 citations. If we considered the abstract to be relevant, we obtained a full copy of the article; we contacted authors if full-text articles could not be retrieved. Furthermore, the reference sections were screened for other eligible citations. We considered 67 articles to be relevant, of which 37 were excluded for the following reasons: 11 were case reports, 7 referred to disseminated NTM infections, 6 were not in English (5 in Japanese, 1 in Chinese), 6 did not concern pulmonary NTM, 4 were reviews, and 3 represented neither epidemiologic data nor clinical cases. From the remaining 30 articles (4-33), the following data were abstracted for this review: country, research setting, NTM species, clinical features of the patients, and radiographic data. All articles were screened to determine whether ATS diagnostic criteria for the determination of clinical relevance of NTM isolations applied (1). Cases consistent with the ATS diagnostic criteria were considered clinically relevant.

\section{Search Results}

We identified 30 English-language articles about the epidemiology and clinical relevance of NTM isolates in Asia (4-33). We found data from China (5), Hong Kong (6), India (7-13), Japan (4,14-20), South Korea (21-24), Singapore (25), Taiwan (26-29), and Thailand (30-33). Most articles used a combination of methods to identify NTM species. Biochemical and phenotypic analysis $(\mathrm{n}=$ 11) were used most frequently, followed by molecular tools $(n=4)$ or a combination of both $(n=5)$. From 10 articles, the exact methods of species identification could not be determined. The use of identification methods differed over time; biochemical and phenotypic analyses were mostly used during 1966-1990 (6 of 7 studies), whereas use of molecular tools increased during 1990-2009 (Table 1). The number of different species isolated did not differ between the 2 periods. However, most species identification by biochemical testing depended heavily on 1 study from India (11).

\section{Epidemiology}

Regardless of clinical relevance, 25 articles reported on NTM isolates from pulmonary samples (Figure 1) (4-13,17-22,25-33). In general, Mycobacterium avium complex (MAC) was most frequently (67\%) isolated, although it predominated in northeastern Asia (South Korea and Japan). Exact species identification of MAC (now $M$. avium, $M$. intracellulare, $M$. chimaera, $M$. colombiense, $M$. vulneris, M. marseillense, M. bouchedurhonense, and $M$. timonense) $(34,35)$ was not performed, thereby hampering a more detailed analysis.

Rapidly growing mycobacteria ([RGM] M. fortuitum complex, M. abscessus, M. chelonae) were frequently identified in pulmonary samples from Taiwan, China, and Singapore $(5,25-29)$. The overall isolation of RGM in this part of Asia was 16\%, making RGM the second most frequently isolated species in this region. Other species frequently encountered were M. kansasii (4\%) and M. gordonae (3.5\%). M. malmoense, a species regularly encountered in northwestern Europe, was found only 9 times in a single study of 1,000 NTM isolates from India (11). Also, M. xenopi, which is frequently encountered in Canada and England (3), was isolated only 10 times (of 11,987 isolates) and mainly during studies from India $(7,11,13,21,27)$.

\section{Clinical Relevance}

The clinical relevance of pulmonary NTM isolates (Table 2) was mentioned in 10 articles covering a total of 1,744 patients $(6,16,21,23,25,28-30,32,33)$. No articles covered the clinical relevance of NTM in China. Different criteria were used to define clinical relevance, but all criteria comprised a combination of clinical, bacteriologic, and radiographic criteria. Only 4 studies reported a priori use of ATS criteria (1). According to ATS criteria, for $31 \%$

\begin{tabular}{|c|c|c|}
\hline Species & $\begin{array}{l}\text { Before } 1990 \\
(\mathrm{n}=1,205) \dagger\end{array}$ & $\begin{array}{c}\text { After 1990 } \\
(\mathrm{n}=7,614) \dagger\end{array}$ \\
\hline Mycobacterium abscessus & - & $\mathrm{x}$ \\
\hline M. aichiense & $x$ & - \\
\hline M. asiaticum & $\mathrm{x}$ & - \\
\hline M. avium complex & $x$ & $x$ \\
\hline M. celatum & - & $\mathrm{x}$ \\
\hline M. chelonae & $x$ & $x$ \\
\hline M. flavescens & $x$ & $x$ \\
\hline M. fortuitum & $x$ & $x$ \\
\hline M. gastri & $x$ & $x$ \\
\hline M. gordonae & $x$ & $x$ \\
\hline$M$ hemophilum & $x$ & - \\
\hline M. kansasii & $\mathrm{x}$ & $\mathrm{x}$ \\
\hline M. malmoense & $x$ & - \\
\hline M. marinum & $\mathrm{x}$ & $\mathrm{x}$ \\
\hline M. neoarum & $x$ & - \\
\hline M. parafortuitum & $x$ & - \\
\hline M. phlei & $x$ & $x$ \\
\hline M. scrofulaceum & $x$ & $x$ \\
\hline M. simiae & $x$ & $x$ \\
\hline M. smegmatis & $x$ & $x$ \\
\hline M. szulgai & $x$ & $x$ \\
\hline M. terrae & $x$ & $x$ \\
\hline M. tokaiense & $x$ & - \\
\hline M. triviale & $x$ & $x$ \\
\hline M. thermophilum & $x$ & - \\
\hline M. thermoresistibile & $\mathrm{x}$ & - \\
\hline M. ulcerans & $x$ & - \\
\hline M. vaccae & $\mathrm{x}$ & $\mathrm{X}$ \\
\hline M. xenopi & $x$ & $\mathrm{x}$ \\
\hline
\end{tabular}




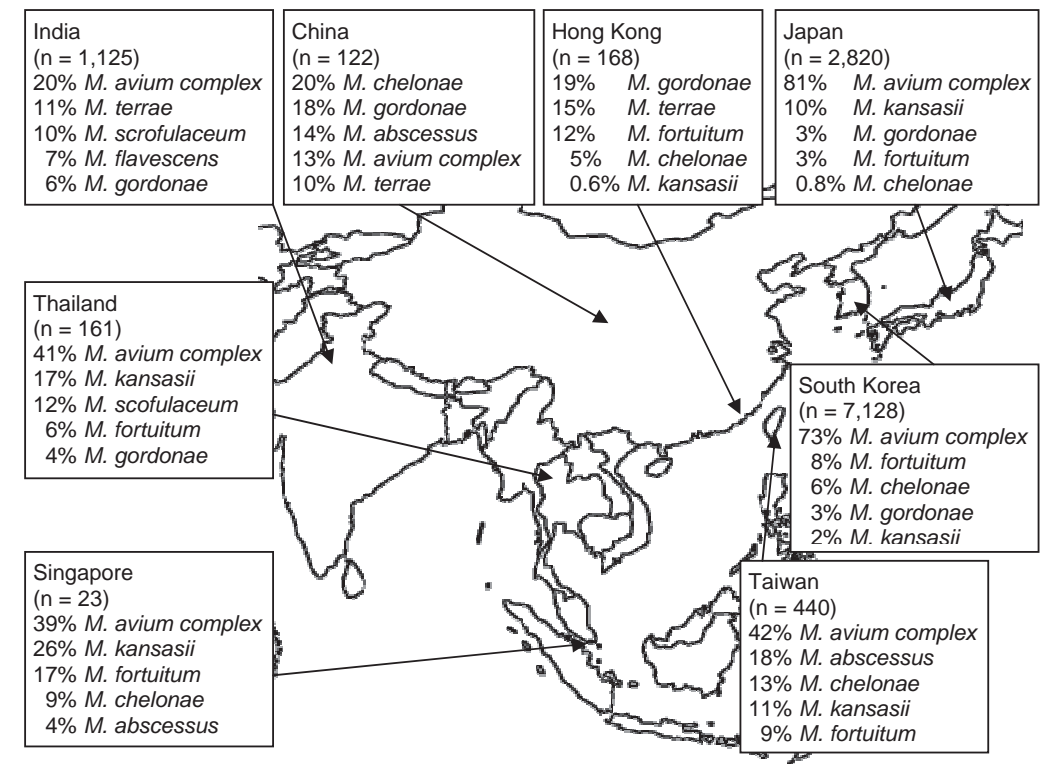

Figure 1. Five most prevalent nontuberculous mycobacteria species found in respiratory specimens, regardless of clinical relevance, Asia, 1971-2007. Data from (4-13,17-22,25-33).

$(582 / 1,744)$ of patients, pulmonary NTM isolates were considered clinically relevant. Relevance varied widely across regions and studies. For example, a study of patients in an intensive care unit in Taiwan found that only $9 \%$ of NTM isolates were clinically relevant (28); however, in a study of HIV-infected patients in Thailand, relevance rose to $76 \%(30)$.

Figure 2 shows the distribution of mycobacterial species among patients with definite pulmonary NTM disease in Asia, according to ATS criteria. MAC was the most frequently reported (68\% of all cases) cause of NTM disease; RGM were second (14\% of all cases). Prevalence of RGM pulmonary infections ranged from $2.6 \%$ (Japan) to $44 \%$ (South Korea). M. malmoense and M. xenopi were not reported as causative species in any of these studies in Asia.

We found some discrepancies between isolation frequency (Figure 1) and clinical relevance (Figure 2). For example, in Hong Kong $M$. gordonae was found in $19 \%$ of pulmonary isolates regardless of clinical relevance but in only $4 \%$ of cases of pulmonary NTM infection. In contrast, in India, higher prevalence of $M$. szulgai was found $(1 \%$ in all pulmonary samples vs. $7 \%$ of all causes of pulmonary NTM). We therefore investigated the clinical relevance of the various NTM species separately, measuring clinical relevance per

\begin{tabular}{|c|c|c|c|c|}
\hline Study area & $\begin{array}{c}\text { No. } \\
\text { patients }\end{array}$ & $\begin{array}{l}\text { Patients for whom NTM } \\
\text { infection was considered } \\
\text { clinically relevant, } \%\end{array}$ & Criteria used (year of revision) & Reference \\
\hline Hong Kong & 168 & 17 & ATS criteria (1990) & (6) \\
\hline Japan & 357 & $76 \dagger$ & ATS criteria (1997) & (16) \\
\hline South Korea & 794 & 17 & ATS criteria (1997) & $(21)$ \\
\hline South Korea & 23 & $65 \ddagger$ & BTS criteria (1999) & (23) \\
\hline Singapore & 23 & 65 & $\begin{array}{c}\text { Compatible signs and symptoms, }>3 \text { positive sputum specimens, } \\
\text { and radiographic features of recent lung disease }\end{array}$ & (25) \\
\hline Taiwan & 111 & 9 & ATS criteria (1990) & (28) \\
\hline Taiwan & 169 & $28 \S$ & $\begin{array}{c}\text { NTM in >2 samples, new radiographic lesions, and no other } \\
\text { pathogens (definite) or other concomitant bacteria (probable NTM) } \\
\text { found }\end{array}$ & (29) \\
\hline Thailand & 33 & 76 & $\begin{array}{l}\text { NTM in sample(s); compatible signs, symptoms, and radiographic } \\
\text { features; and no other explanation }\end{array}$ & (30) \\
\hline Thailand & 24 & 29 & $\begin{array}{c}\text { Continued NTM isolation, progressive pulmonary disease, and } \\
\text { worsening radiographic lesions }\end{array}$ & (32) \\
\hline Thailand & 42 & 71 & $\begin{array}{l}\text { Repeated isolation of high numbers of NTM and presence of } \\
\text { compatible disease process }\end{array}$ & (33) \\
\hline
\end{tabular}

${ }^{*}$ NTM, nontuberculous mycobacteria; ATS, American Thoracic Society (1); BTS, British Thoracic Society.

†Only Mycobacterium avium complex bacteria were included in this study.

‡Only M. kansasii was included.

$\S 14 \%$ if only definite NTM were included. 


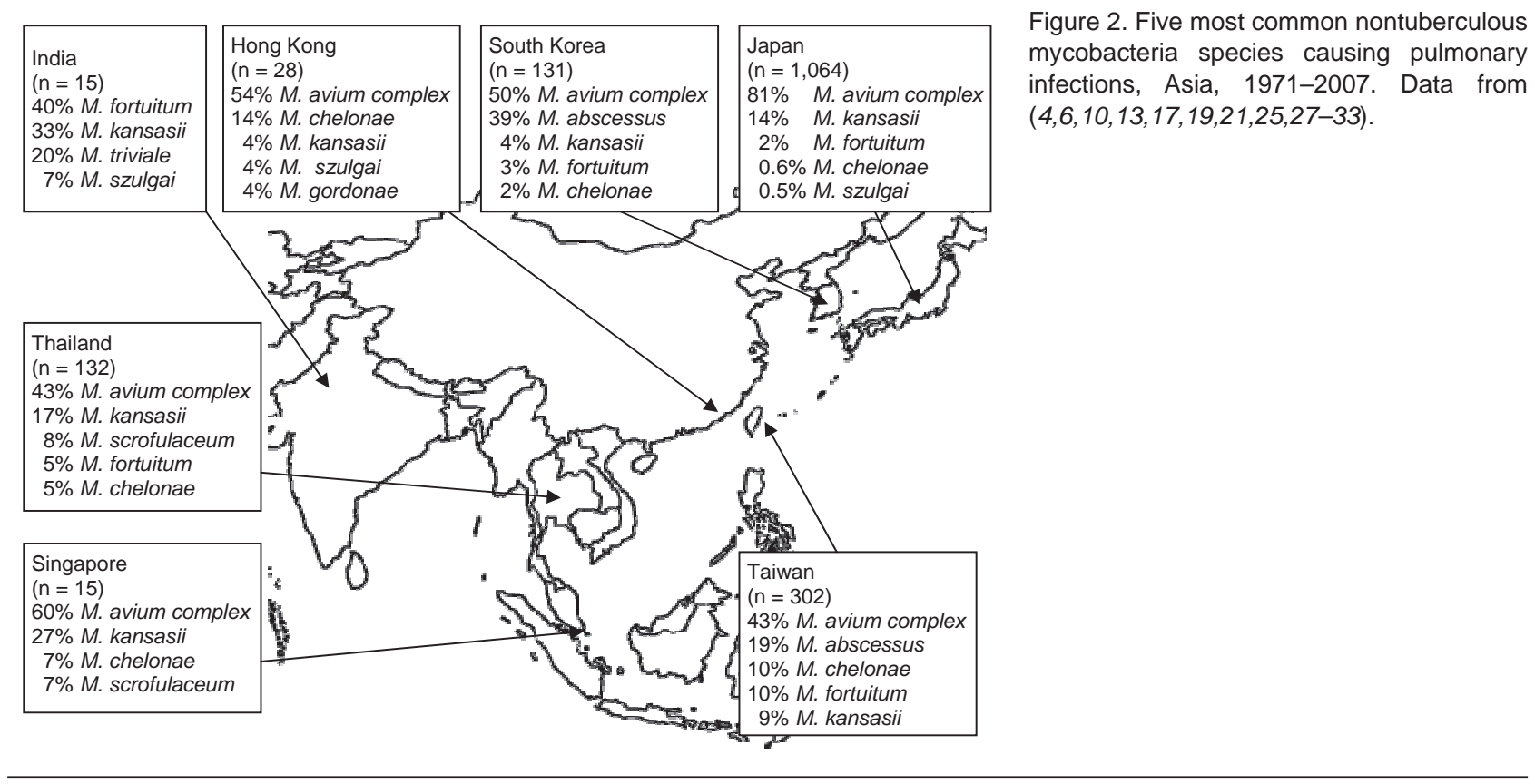

species by the percentage of patients meeting ATS criteria (Figure 3). Most clinically relevant species were MAC (56\%), followed by M. abscessus (35\%) and M. chelonae (31\%). M. fortuitum, M. gordonae, and M. terrae were infrequently reported $(1 \%-3 \%)$ as clinically relevant. The varying clinical relevance per species might partially explain the differences between Figure 1 and Figure 2.

\section{Clinical Signs and Radiographic Features}

We found information on clinical signs for 689 patients and radiographic data for 1,044 patients $(10,12,14$ 16,20,21,23-25,27-30,33). Most patients with pulmonary NTM infections were male (543/689). Because of incomplete data, mean age could not be calculated; 8 studies reported mean ages of 50-70 years. Other characteristics are shown in Table 3 . One third of patients had a history of tuberculosis (TB) (252/689). HIV co-infection was less prevalent among patients with localized pulmonary NTM infections (15/280). Clinical signs of NTM disease mimicked those typical of TB: most frequently chronic cough (255/268), followed by hemoptysis (82/268), fever (47/268), and weight loss (40/268). Radiographically, most patients had structural lung disease; 39\% $(405 / 1,044)$ had cavitations and 44\% $(461 / 1,044)$ had bronchiectasis.

\section{Discussion}

NTM isolation and disease in Asia have several features. First, a substantial percentage $(31 \%)$ of patients from whom pulmonary NTM were isolated had clinically relevant NTM disease. This finding is similar to the $33 \%$ and $25 \%$ found in studies in Canada and the Netherlands, respectively $(2,3)$. In Asia, NTM may cause substantial pulmonary disease; differences in clinical relevance exist among species (Figure 3), as previously observed (2).

Second, MAC was the main cause of pulmonary NTM infection (68\% of cases) in Asia. In a key article in 2002, Marras and Daley reviewed the prevalence of pulmonary NTM disease in the world (3). They noted a predominance of MAC among the causative agents of pulmonary NTM disease in Asia. Data from Asia were, however, scarce, and their conclusion was mainly based on 1 study (6). Our

\begin{tabular}{lc}
\hline Table 3. Clinical and radiographic characteristics for patients with \\
pulmonary nontuberculous mycobacteria infections in Asia, \\
1971-2007* & No. (\%) patients \\
\hline Characteristic & $79(11)$ \\
\hline Concurrent conditions, $\mathrm{n}=689$ & $40(6)$ \\
Malignancy, hematologic or solid & $15(5)$ \\
Gastrointestinal disease & $18(3)$ \\
HIV infection, $\mathrm{n}=280$ tested & $18(3)$ \\
Chronic corticosteroid treatment & $13(2)$ \\
Diabetes mellitus & \\
Renal disease & $252(37)$ \\
\hline Previous lung disease, $\mathrm{n}=689$ & $62(9)$ \\
Tuberculosis & $47(7)$ \\
Chronic obstructive pulmonary disease & \\
Bronchiectasis & $255(95)$ \\
\hline Clinical signs, $\mathrm{n}=268$ & $82(31)$ \\
Chronic cough & $47(18)$ \\
Hemoptysis & $40(15)$ \\
Fever & $405(39)$ \\
Weight loss & $559(54)$ \\
\hline Radiographic findings, $\mathrm{n}=1,044$ & $461(44)$ \\
Cavitation & \\
Nodular & \\
Bronchiectasis & \\
\hline Data from (10,12,14-16,20,21,23-25,27-30,33). & \\
\hline & \\
\hline
\end{tabular}




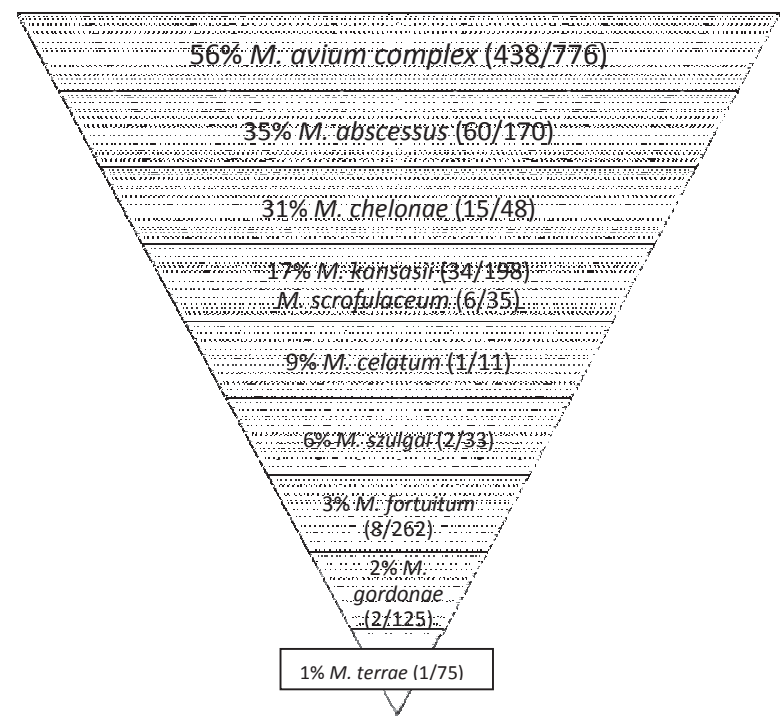

Figure 3. Clinical relevance of pulmonary nontuberculous mycobacterium (NTM) isolates, Asia, 1971-2007. Relevance per species was defined as percentage of patients with pulmonary NTM isolates meeting the American Thoracic Society criteria. Species reported infrequently, i.e., $<5 \times$, are not shown. Data from $(6,16,17,21,23,25,29,32,33)$.

study supports their conclusion of the predominance of MAC in Asia, which is consistent with its predominance in other parts of the world, namely, North America and most parts of Europe (3).

Third, we found that in some regions in Asia, RGM are a major cause of pulmonary NTM disease. This finding contrasts with studies of NTM in other parts of the world (3). In a surveillance study from the Netherlands for instance, RGM caused only $3 \%$ of all pulmonary NTM infections (2). In the United States, this percentage is $\approx 5 \%$ (36). In the present review, RGM was found to generally cause $14 \%$ of pulmonary NTM infections, but in 3 countries (India, Taiwan, South Korea) this percentage rose to $>30 \%$ of infections. The fact that RGM were frequently found in pulmonary samples (Figure 1) could reflect higher environmental exposure of RGM in Asia and, hence, higher isolation frequency. The predominance of RGM species may be the result of laboratory practices as well. Ethnic factors may also contribute to susceptibility to different species; i.e., Asian persons could be more susceptible to RGM infection.

Contrary to the high frequency of isolation of RGM species, M. malmoense and $M$. xenopi, frequently seen in other parts of the world, were not seen as causative species in any of the studies from Asia. M. xenopi has been associated with hot water systems (1); as a result, it might be expected to be more rare in Asia, where the water delivery infrastructure is less developed than that in Europe and North America.

A fourth feature of pulmonary NTM disease in Asiacompared with Europe and North America-was the relatively high percentage of patients with a history of TB. This finding might merely reflect the higher incidence of TB in Asia, or it could reflect higher clinician awareness in Asia, such that physicians order Mycobacterium spp. cultures in former TB patients with coughing and hence find a relatively higher number of NTM isolates. Alternatively, it could reflect a true predilection of NTM for patients with structural lung disease (1) associated with a higher susceptibility to mycobacterial infection in general. The role of TB in the pathogenesis of pulmonary NTM disease is controversial; structural lung damage by a TB infection renders the host vulnerable to NTM disease (1), but there are also clues that exposure to TB infers cross-protection to NTM disease (37).

Our study has some limitations. The major limitation is the language restriction. The inclusion of languages other than English would probably have increased precision. For instance, during our literature search we came across 5 articles, published as abstracts in PubMed, on NTM infections in Japan. Although certain aspects of these data were already published in the English-language articles we included, we did not have the means to include these non-English-language studies. Nevertheless, our study illustrates the distribution of NTM infections from different geographic areas in Asia and will increase knowledge of the distribution and relevance of NTM species in Asia.

Another limitation is the long time span of the included studies. Because they ranged from 1969 to 2008, they used different decontamination, culture, and identification methods. Data should therefore be considered with caution because of the variety of laboratory procedures used by the several authors to isolate and identify NTM. First, different sample decontamination protocols may determine the yield of NTM by selecting for certain NTM species and inhibiting others (38). Second, the introduction of more sensitive liquid media probably increased NTM isolation and perhaps selected for specific species (26). Finally, and foremost, the major differences in identification methods used in the studies introduce important biases. Because the taxonomy of NTM has been changing in recent years $(37,39,40)$, the different NTM identification methods used in the various studies might have influenced our results; use of molecular tools to identify the historical isolates would probably result in different, more detailed, identifications, especially among the MAC, the M. simiae group, and the $\operatorname{RGM}(37,39,40)$. We did note increased use of molecular tools for NTM identification over time (Table 1), which will, over the next few years, provide us with a more up-todate overview of NTM species distribution in Asia. 
In conclusion, despite the limitations of language and species identification methods, we have described the scale of human pulmonary NTM infections in eastern Asia. MAC bacteria were not only the most prevalent NTM found in pulmonary samples, but they were also the most common cause of pulmonary NTM disease in this geographic region. Distinct epidemiologic and clinical characteristics of pulmonary NTM disease in Asia were found: many patients had a history of TB, and RGM were a frequent cause of pulmonary NTM infections. These distinct characteristics of pulmonary NTM disease in this part of the world are not well reflected in the current ATS criteria on NTM infections and could be incorporated in future guidelines.

Dr Simons is a resident in pulmonary medicine at the Radboud University Nijmegen Medical Centre. His primary research interest focuses on resistance mechanisms in multidrugresistant tuberculosis.

\section{References}

1. Griffith DE, Aksamit T, Brown-Elliot BA, Catanzaro A, Daley C, Gordin F, et al. An official ATS/IDSA statement: diagnosis, treatment and prevention of nontuberculous mycobacterial diseases. Am J Respir Crit Care Med. 2007;175:367-416. DOI: 10.1164/ rccm.200604-571ST

2. van Ingen J, Bendien SA, de Lange WC, Hoefsloot W, Dekhuijzen PN, Boeree MJ, et al. Clinical relevance of non-tuberculous mycobacteria isolated in the Nijmegen-Arnhem region, the Netherlands. Thorax. 2009;64:502-6. DOI: 10.1136/thx.2008.110957

3. Marras TK, Daley C. Epidemiology of human pulmonary infection with nontuberculous mycobacteria. Clin Chest Med. 2002;23:55367. DOI: 10.1016/S0272-5231(02)00019-9

4. Tsukamura M, Shimoide H, Kita N, Kawakami K, Ito T, Nakajima $\mathrm{N}$, et al. Epidemiologic studies of lung disease due to mycobacteria other than Mycobacterium tuberculosis in Japan. Rev Infect Dis. 1981;3:997-1007.

5. Weimin L, Guanglu J, Zhihui L, Huakan H, Liquan C, Miao T, et al. Non-tuberculous mycobacteria in China. Scand J Infect Dis. 2007;39:138-41. DOI: 10.1080/00365540600951234

6. Hosker HSR, Lam CW, Ng TK, Ma HK, Chan SL. The prevalence and clinical significance of pulmonary infection due to non-tuberculous mycobacteria in Hong Kong. Respir Med. 1995;89:3-8. DOI: 10.1016/0954-6111(95)90063-2

7. Agarwal SK, Jamil Z, Srivasta VK, Rathi AK, Mehrotra L. A clinico-bacteriological assessment of pulmonary tuberculosis patients with reference to drug resistance pattern in treated and untreated cases. Indian J Med Sci. 1980;34:39-46.

8. Choudri DS, Dube MK, Purohit SD, Dube S. The prevalence of anonymous mycobacteria in both resistant as well as fresh cases of pulmonary tuberculosis in the local population of south east Rajasthan. Indian J Pathol Microbiol. 1979;22:165-75.

9. Jesudason MV, Gladstone P. Non tuberculous mycobacteria isolated from clinical specimens at a tertiary care hospital in south India. Indian J Med Microbiol. 2005;23:172-5. DOI: 10.4103/02550857.16589

10. Karak K, Bhattacharyya S, Majumdar S, De PK. Pulmonary infection caused by mycobacteria other than M. tuberculosis in and around Calcutta. Indian J Pathol Microbiol. 1996;39:131-4.
11. Paramasivan CN, Govindan D, Prabhakar R, Somasundaram PR, Subbammal S, Tripathy SP. Species level identification of nontuberculous mycobacteria from south Indian BCG trial area during 1981. Tubercle. 1985;66:9-15. DOI: 10.1016/0041-3879(85) 90048-0

12. Ramakrishnan CV. Pulmonary disease due to atypical mycobacteria: a retrospective study from south India. Rev Infect Dis. 1981;3: 1090-2.

13. Shanker SVR, Jain NK, Chandrasekhar S, Singh MM. Prevalence of atypical mycobacteria in sputum of patients undergoing treatment at a tuberculosis clinic. Indian J Chest Dis Allied Sci. 1989;31:9-13.

14. Kobashi Y, Matsushima T. Comparison of clinical features in patients with pulmonary Mycobacterium-avium complex (MAC) disease treated before and after proposal for guidelines. J Infect Chemother. 2004;10:25-30. DOI: 10.1007/s10156-003-0273-3

15. Kobashi Y, Matsushima T. The microbiological and clinical effects of combined therapy according to guidelines on the treatment of pulmonary Mycobacterium avium complex disease in Japan-including a follow-up study. Respiration. 2007;74:394-400. DOI: $10.1159 / 000095674$

16. Okumura M, Iwai K, Ogata H, Ueyama M, Kubota M, Aoki M, et al. Clinical factors on cavitary and nodular bronchiectatic types in pulmonary Mycobacterium avium complex disease. Intern Med. 2008;47:1465-72. DOI: 10.2169/internalmedicine.47.1114

17. Saito H, Watanabe T, Akamatsu S, Yamamoto Y, Irikura T, Kubonishi $\mathrm{K}$, et al. Incidence of atypical mycobacteria and pulmonary mycobacterioses in national sanatoria in the Chugoku-Shikoku area. Hiroshima J Med Sci. 1979;28:161-5.

18. Rapid increase of the incidence of lung disease due to Mycobacterium kansasii in Japan. Chest. 1983;83:890-2. DOI: 10.1378/ chest.83.6.890

19. Tsukamura M, Kita N, Shimoide H, Arakawa H, Kuze A. Studies on the epidemiology of nontuberculous mycobacteriosis in Japan. Am Rev Respir Dis. 1988;137:1280-4.

20. Yamamoto M. Pulmonary disease due to Mycobacterium szulgai in Japan. Intern Med. 2000;39:277-9. DOI: 10.2169/internalmedicine.39.277

21. Koh WJ, Kwon OJ, Jeon K, Kim TS, Lee KS, Park YK, et al. Clinical significance of nontuberculous mycobacteria isolated from respiratory specimens in Korea. Chest. 2006;129:341-8. DOI: 10.1378/ chest.129.2.341

22. Ryoo SW, Shin S, Shim MS, Park YS, Lew WJ, Park SN, et al. Spread of nontuberculous mycobacteria from 1992 to 2006 in Koreans. J Clin Lab Anal. 2008;22:415-20. DOI: 10.1002/jcla.20278

23. Yim JJ, Park YK, Lew WJ, Bai GH, Han SK, Shim YS. Mycobacterium kansasii pulmonary disease in Korea. J Korean Med Sci. 2005;20:957-60. DOI: 10.3346/jkms.2005.20.6.957

24. Yim JJ, Kim HJ, Kwon OJ, Koh WJ. Association between microsatellite polymorphisms in intron II of the human Toll-like receptor 2 gene and nontuberculous mycobacterial lung disease in a Korean population. Hum Immunol. 2008;69:572-6. DOI: 10.1016/j. humimm.2008.06.003

25. Teo SK, Lo KL. Nontuberculous mycobacterial disease of the lungs in Singapore. Singapore Med J. 1992;33:464-6.

26. Chien HP, Yu MC, WU MH, Lin TP, Luh KT. Comparison of the BACTEC MGIT 960 with Löwenstein-Jensen medium for recovery of mycobacteria from clinical specimens. Int J Tuberc Lung Dis. 2000;4:866-70.

27. Ding LW, Lai CC, Lee LN, Hsueh PR. Disease caused by nontuberculous mycobacteria in a university hospital in Taiwan, 1997-2003. Epidemiol Infect. 2006;134:1060-7. DOI: 10.1017/ S0950268805005698

28. Shih JY, Hsueh PR, Lee LN, Wang HC, Yang PC, Kuo SH, et al. Nontuberculous mycobacteria isolates: clinical significance and disease spectrum. J Formos Med Assoc. 1997;96:621-7. 
29. Shu CC, Lee CH, Wang JY, Jerng JS, Yu CJ, Hsueh PR, et al. Nontuberculous mycobacteria pulmonary infection in medical intensive care unit: the incidence, patient characteristics, and clinical significance. Intensive Care Med. 2008;34:2194-201. DOI: 10.1007/ s00134-008-1221-6

30. Ratanasuwan W, Techasathit W, Chuenarom V, Suwanagool S, Anekthananont T, Jearanaisilavong J, et al. Infection due to nontuberculous Mycobacterium other than MAC in AIDS patients at Siriraj Hospital during 1998-2000: saphophyte vs pathogen. J Med Assoc Thai. 2002;85:886-93.

31. Saritsiri S, Udomsantisook N, Suankratay C. Nontuberculous infections in King Chulalongkorn Memorial Hospital. J Med Assoc Thai. 2006;89:2035-46.

32. Sriyabhaya N, Wongwatana S. Pulmonary infection caused by atypical mycobacteria: a report of 24 cases in Thailand. Rev Infect Dis. 1981;3:1085-9

33. Wongwatana S, Sriyabhaya N. Nontuberculous mycobacterial infection of the lung in a chest hospital in Thailand. J Med Assoc Thai. 1992; 75:1-10.

34. van Ingen J, Boeree MJ, Kösters K, Wieland A, Tortoli E, Dekhuijzen PN, et al. Proposal to elevate Mycobacterium avium complex ITS sequevar MAC-Q to Mycobacterium vulneris sp. nov. Int J Syst Evol Microbiol. 2009;59:2277-82. DOI: 10.1099/ijs.0.008854-0

35. Ben Salah I, Cayrou C, Raoult D, Drancourt M. Mycobacterium marseillense sp. nov., Mycobacterium timonense sp. nov., and Mycobacterium bouchedurhonense sp. nov., members of the Mycobacterium avium complex. Int J Syst Evol Microbiol. 2009;59:2803-8. DOI: $10.1099 /$ ijs.0.010637-0
36. O'Brien RJ, Geiter LJ, Snider D. The epidemiology of nontuberculous mycobacterial diseased in the United States. Am Rev Respir Dis. 1987; 135:1007-14

37. Trnka L, Dankova D, Svandova E. Six years' experience with the discontinuation of BCG vaccination: 4. Protective effect of BCG vaccination against the Mycobacterium avium intracellulare complex. Tuber Lung Dis. 1994;75:348-52. DOI: 10.1016/09628479(94)90080-9

38. Buijtels PC, Petit PL. Comparison of $\mathrm{NaOH}-n$-acetyl cysteine and sulfuric acid decontamination methods for recovery of mycobacteria from clinical specimens. J Microbiol Methods. 2005;62:83-8. DOI: 10.1016/j.mimet.2005.01.010

39. Adékambi T, Colson P, Drancourt M. rpoB-based identification of nonpigmented and late-pigmenting rapidly growing mycobacteria. J Clin Microbiol. 2003;41:5699-708. DOI: 10.1128/JCM.41.12.56995708.2003

40. Tortoli E. Impact of genotypic studies on mycobacterial taxonomy: the new mycobacteria of the 1990s. Clin Microbiol Rev. 2003;16:319-54. DOI: 10.1128/CMR.16.2.319-354.2003

Address for correspondence: Sami Simons, Radboud University Medical Centre Nijmegen, Department of Pulmonary Diseases-454, PO Box 9101, 6500 HB Nijmegen, the Netherlands; email: s.simons@long.umcn.nl

Use of trade names is for identification only and does not imply endorsement by the Public Health Service or by the US Department of Health and Human Services.

Email:
eideditor@ @cdc.gov
Fax:
404-639-1954
Mail:
CDC/MS D61
1600 Clifton Rd NE
Atlanta, GA 30333
USA

